\title{
Structural Uniformity of Pullulan Produced by Several Strains of Pullularia pullulans ${ }^{\dagger}$
}

\author{
Ryo Taguchi, * Yo Kikuchi, Yoshiyuki SaKano and Tsuneo Kobayashi \\ Department of Agricultural Chemistry, Tokyo Noko University, Fuchu, Tokyo, 183 \\ Received November 4, 1972
}

\begin{abstract}
Extracellular polysaccharides produced by 3 strains of Pullularia pullulans were fractionated by treating with cetyl trimethylammonium hydroxide into soluble and insoluble fractions, and the structure of the former fraction, i.e., pullulan, was studied. The yield and the ratio of 2 fractions varied widely according to the strains. But the structure of pullulan was found to be uniform irrespective of the strains used. All 3 samples of pullulan gave only glucose on complete acid hydrolysis, and $93 \sim 95 \%$ maltotriose and $5 \sim 7 \%$ maltotetraose after isoamylase (pullulanase) action. The ratio of $a-1,4-$ to $a-1,6$-glucosidic linkages calculated from periodate oxidation data coincided very well with the value expected from the ratio of maltotriose to maltotetraose units. An evidence for the complete absence of branch structure in pullulan was presented from the results of hydrolysis by pullulan 4-glucanohydrolase.
\end{abstract}

Pullulan, a polysaccharide produced by a fungus in the Genus Pullularia, mainly consists of maltotriose units joined through $a$ 1,6-glucosidic linkages. ${ }^{1 \sim 4)}$ But it was not certain whether the pullulan produced by various strains of organism is uniform in structure. Ueda et $a l^{4}{ }^{4}$ have reported that the structure of polysaccharide obtained by several strains of Pullularia differs considerably as judged from results of periodate oxidation and susceptibility to crystalline glucoamylase. Recently Catley and Whelan ${ }^{5}$ found $6.6 \%$ of maltotetraosyl units in pullulan produced by a strain, and assumed that the structure of pullulan might be varied in each strain of organism.

The authors have intended to study the biosynthetic mechanism of pullulan. As the first step for this purpose, it is required to establish that the structure of pullulan be uniform or not, depending on the strain used. Several samples of crude pullulan from different strains showed considerably different results, when tested with periodate oxidation and degradation by Aerobacter isoamylase ${ }^{1)}$ (pul-

$\uparrow$ Polysaccharide Production by Pullularia pullulans. Part I.

* Present address: Faculty of Pharmaceutical Sciences, Nagoya City University, Mizuho-ku, Nagoya. lulanase, amylopectin 6-glucanohydrolase, EC 3.2. 1.9). But after purification with cetyl trimethylammonium hydroxide (CTA) treatment, ${ }^{6)}$ pullulan from several strains gave essentially the same results on acid hydrolysis, periodate oxidation, as well as on degradation by Aerobacter isoamylase and pullulan 4glucanohydrolase $(\alpha-1,4$-pullulanase $\left.){ }^{7}\right)$ the last-named enzyme being recently discovered and characterized in our laboratory.

\section{MATERIALS AND METHODS}

1. Microorganism. Three strains of Pullularia pullulans, S-1, OUT 4242 and IFO 6353, were used as polysaccharide producers. The strain $S-1$ was isolated and kindly supplied by Dr. S. Ueda, Kyushu University.

2. Medium and cultivation. Stock medium contained sucrose $2 \%$, yeast extract $0.4 \%, \mathrm{~K}_{2} \mathrm{HPO}_{4} 0.3 \%$, $\mathrm{NaCl} 0.1 \%,\left(\mathrm{NH}_{4}\right)_{2} \mathrm{SO}_{4} 0.06 \%, \mathrm{MgSO}_{4} \cdot 7 \mathrm{H}_{2} \mathrm{O} 0.02 \%$, FeSO$\cdot 7 \mathrm{H}_{2} \mathrm{O} \quad 0.01 \%$ and agar $1.5 \%$. Organism grown on slants containing stock medium at $30^{\circ} \mathrm{C}$ for $24 \mathrm{hr}$ was stored at $5^{\circ} \mathrm{C}$. Spores on stock culture were transferred to the same medium containing sucrose $5 \%$, cultured for $48 \mathrm{hr}$ at $30^{\circ} \mathrm{C}$, and then transferred into $100 \mathrm{ml}$ of liquid medium containing sucrose $5 \%$ and other ingredients except agar in $500 \mathrm{ml}$ shaking fiasks. Inoculated media were shaken at $30^{\circ} \mathrm{C}$.

3. Separation and purification of extracellular polysaccharides. Cultured broth after $96 \mathrm{hr}$ of 
shaking was centrifuged at $8000 \times g$ for $10 \mathrm{~min}$ to remove fungal cells, and to the supernatant 1.5 times its volume of acetone was added under vigorous stirring. The polysaccharide fraction precipitated was dissolved in hot water, and reprecipitated with $50 \%$ ethanol. The precipitate was dissolved in water, and fractionated by adding $0.1 \%$ CTA ethanol solution $^{6}$ at $\mathrm{pH} 5 \sim 6$ into soluble and precipitated fractions. The soluble fraction corresponding to pullulan was repeatedly boiled in $90 \%$ methanol under reflux, centrifuged, washed with ether, and dried in vacuo. This is designated as purified pullulan.

4. Acid hydrolysis. Ten $\mathrm{mg}$ of pullulan was hydrolyzed in $1 \mathrm{ml} 0.5 \mathrm{~N}$ or $1 \mathrm{~N} \mathrm{HCl}$ at $100^{\circ} \mathrm{C}$ for $15 \sim$ $120 \mathrm{~min}$, deionized with ion-exchange resin, and sugars in the hydrolyzate were analyzed with paper chromatography.

5. Enzymatic hydrolysis. Aerobacter isoamylase (pullulanase) free from $a$-amylase and glucoamylase activities was prepared from Aerobacter aerogenes. ${ }^{11}$ Pullulan 4-glucanohydrolase was prepared as described by Sakano et al. ${ }^{7}$ from Aspergillus niger. Pullulan samples $(10 \mathrm{mg})$ were separately treated with excess amounts of both enzymes at $40^{\circ} \mathrm{C}$ for $2 \mathrm{hr}$.

6. Periodate oxidation. One hundred $\mathrm{mg}$ each of pullulan samples was oxidized with sodium metaperiodate solution at $\mathrm{pH} 5.0$ in the dark for $96 \mathrm{hr}$. The periodate consumption was estimated with the method of Aspinall and Ferrier, ${ }^{8}$ and formic acid liberation was determined by titration with $0.01 \mathrm{~N}$ $\mathrm{NaOH}$ under nitrogen gas.

7. Determination of carbohydrates. Total carbohydrates were estimated by phenol-sulfuric acid method, ${ }^{9}$ ' and reducing sugars were determined by Nelson-Somogyi method. ${ }^{10,11}$,

8. Paper chromatography. Paper chromatography of sugars was done on Toyo filter paper No. 50 with descending method using the solvent $n$-butanolpyridine-water $(6: 4: 3)$. Chromatograms were treated with glucoamylase by the method of Kainuma and French, ${ }^{12}$, and localization of sugars was detected with silver nitrate-acetone reagent. A series of malto-oligosaccharides used as standards in paper chromatography was prepared from cyclohexaamylose and glucose by coupling reaction of Bacillus macerans amylase according to the method of French et al. ${ }^{13}$ )

9. Viscosity measurement. Viscosity of pullulan was measured in aqueous solutions in an Ostwaldtype viscometer at $25^{\circ} \mathrm{C}$, and intrinsic viscosity was estimated by extrapolating specific viscosity to the concentration zero.

\section{RESULTS}

\section{Yield and composition of polysaccharide}

The yield of total polysaccharide and the ratio of CTA-soluble and CTA-insoluble fractions, produced by 3 strains of organism, are shown in Table I. As shown in the Table, the yield and composition of polysaccharide varied considerably for each strain.

Table I. Yields and Composition of Extracellular Polysaccharide from Several Strains of $P$. pullulans

\begin{tabular}{lcccc}
\hline $\begin{array}{c}\text { Strains } \\
\text { of } \\
\text { organism }\end{array}$ & $\begin{array}{c}\text { Yields of } \\
\text { polysaccharide } \\
\text { (g per 1 broth) }\end{array}$ & $\begin{array}{c}\text { Ratio of two } \\
\text { fractions } \\
(\%)\end{array}$ \\
\cline { 2 - 5 } & $\begin{array}{c}\text { CTA- } \\
\text { sol. } \\
\text { fraction }\end{array}$ & $\begin{array}{c}\text { CTA- } \\
\text { insol. } \\
\text { fraction }\end{array}$ & $\begin{array}{c}\text { CTA- } \\
\text { sol. } \\
\text { fraction }\end{array}$ & $\begin{array}{c}\text { CTA- } \\
\text { insol. } \\
\text { fraction }\end{array}$ \\
\hline S-1 & 21.6 & 2.4 & 90 & 10 \\
IFO 6343 & 0.41 & 0.84 & 33 & 67 \\
OUT 4242 & 0.29 & 0.83 & 26 & 74 \\
\hline
\end{tabular}

\section{Acid hydrolysis}

On complete acid hydrolysis by $1 \mathrm{~N} \mathrm{HCl}$ at $100^{\circ} \mathrm{C}$ for $120 \mathrm{~min}$, all specimens of purified pullulan gave only glucose. On partial hydrolysis by $0.5 \mathrm{~N} \mathrm{HCl}$ at $100^{\circ} \mathrm{C}$ for $15 \mathrm{~min}$, they gave glucose, isomaltose and panose (or isopanose) as major products together with small amounts of maltose and other oligosaccharides. But isomaltotriose was not detected in the hydrolyzate.

\section{Enzymatic hydrolysis}

1) Hydrolysis by isoamylase (pullulanase). Hydrolysis by Aerobacter isoamylase of pullulan was almost complete after $120 \mathrm{~min}$, and large amount of maltotriose with small amount of maltotetraose were detected in the hydrolyzate. Spots corresponding to each saccharide were extracted from chromatograms with boiling water, and determined with phenolsulfuric acid method. Results indicated that all 3 samples of purified pullulan gave 93 $95 \%$ maltotriose, and 5 7\% maltotetraose. Hydrolysis by isoamylase for $5 \mathrm{~min}$ gave spots corresponding to maltotriose, a hexasaccharide and a nonasaccharide, together 


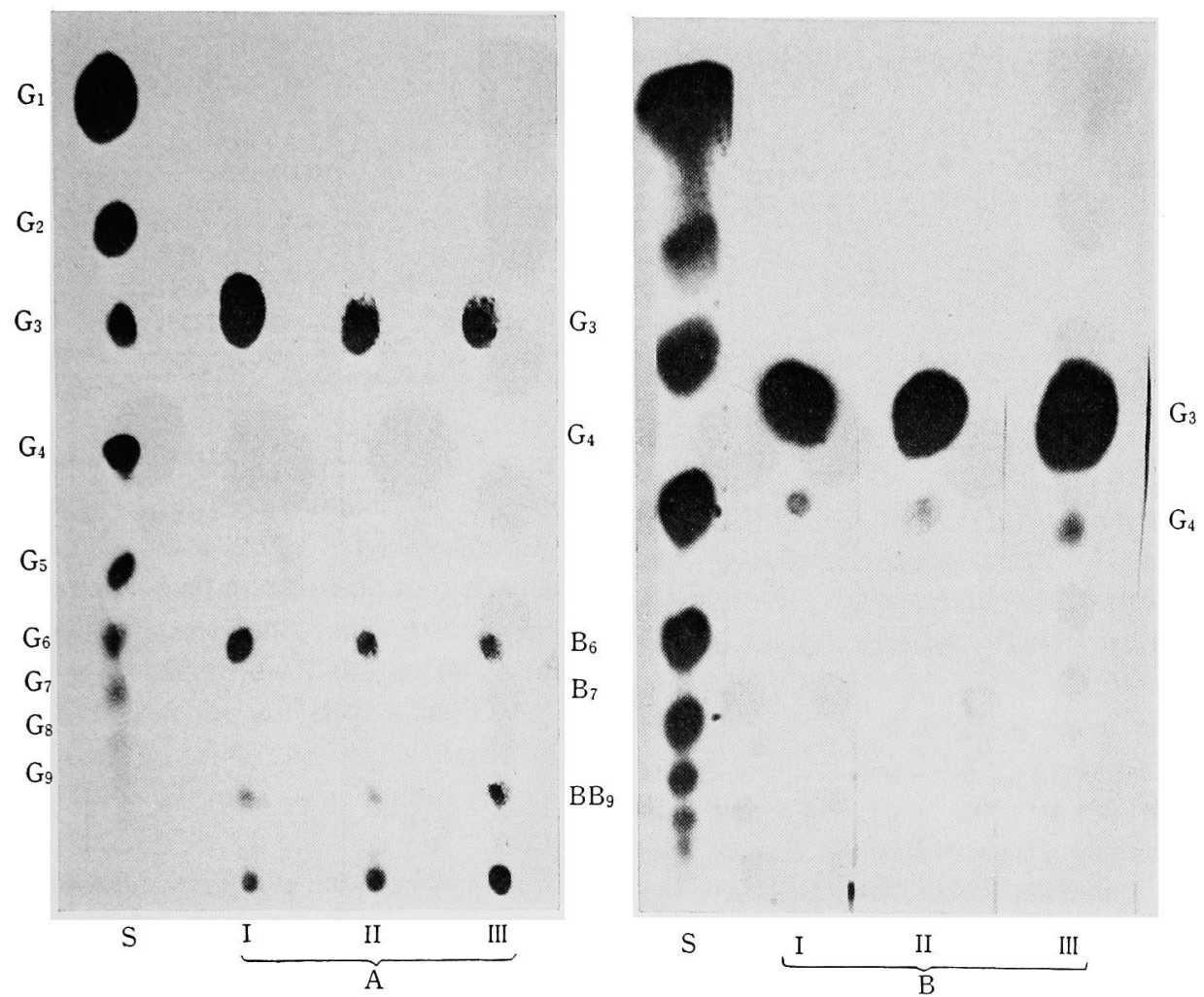

FIG. 1. Hydrolysis of Pullulan by Aerobacter Isoamylase (Pullulanase).

A. Paper chromatogram of partially hydrolyzed pullulan (5 min reaction).

B. Paper chromatogram of completely hydrolyzed pullulan (120 min reaction).

Key: S, standards; $\mathrm{G}_{1} \sim \mathrm{G}_{9}$, glucose $\sim$ maltononaose; I, P. pullulans $\mathrm{S}-1$; II,

$P$. pullulans OUT 4242; III, $P$. pullulans IFO 6353 .

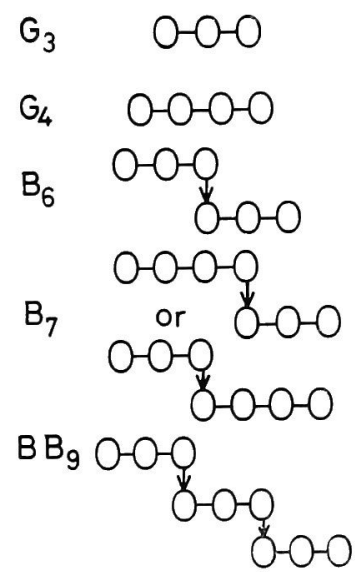

FIG. 2. Structure of Oligosaccharides Formed from Pullulan by Aerobacter Isoamylase (pullulanase) Action.

Key: O, glucose unit; - $-a-1,4$-glucosidic bond; $\downarrow, a$-1,6-glucosidic bond. with faint spots corresponding to maltotetraose and a heptasaccharide. Spots corresponding to hexa-, hepta- and nonasaccharides $\left(\mathrm{B}_{6}, \mathbf{B}_{7}\right.$ and $\mathrm{BB}_{9}$ in Fig. 1) were extracted and subjected to further action of isoamylase. On chromatography after hydrolysis, it was revealed that the hexa- and nonasaccharides gave maltotriose, and the heptasaccharide gave a mixture of maltotriose and maltotetraose. Thus, the structures of these saccharides were assumed to be those given in Fig. 2.

2) Hydrolysis by pullulan 4-glucanohydrolase (a-1,4-pullulanase). This enzyme has been proved $^{7}$ to attack $a-1,4$-glucosidic bonds at the reducing end sides adjacent to $a$-1,6-bonds in pullulan. Hydrolysis of purified pullulan by this enzyme gave saccharides of the polymerization degree 3,6 and 

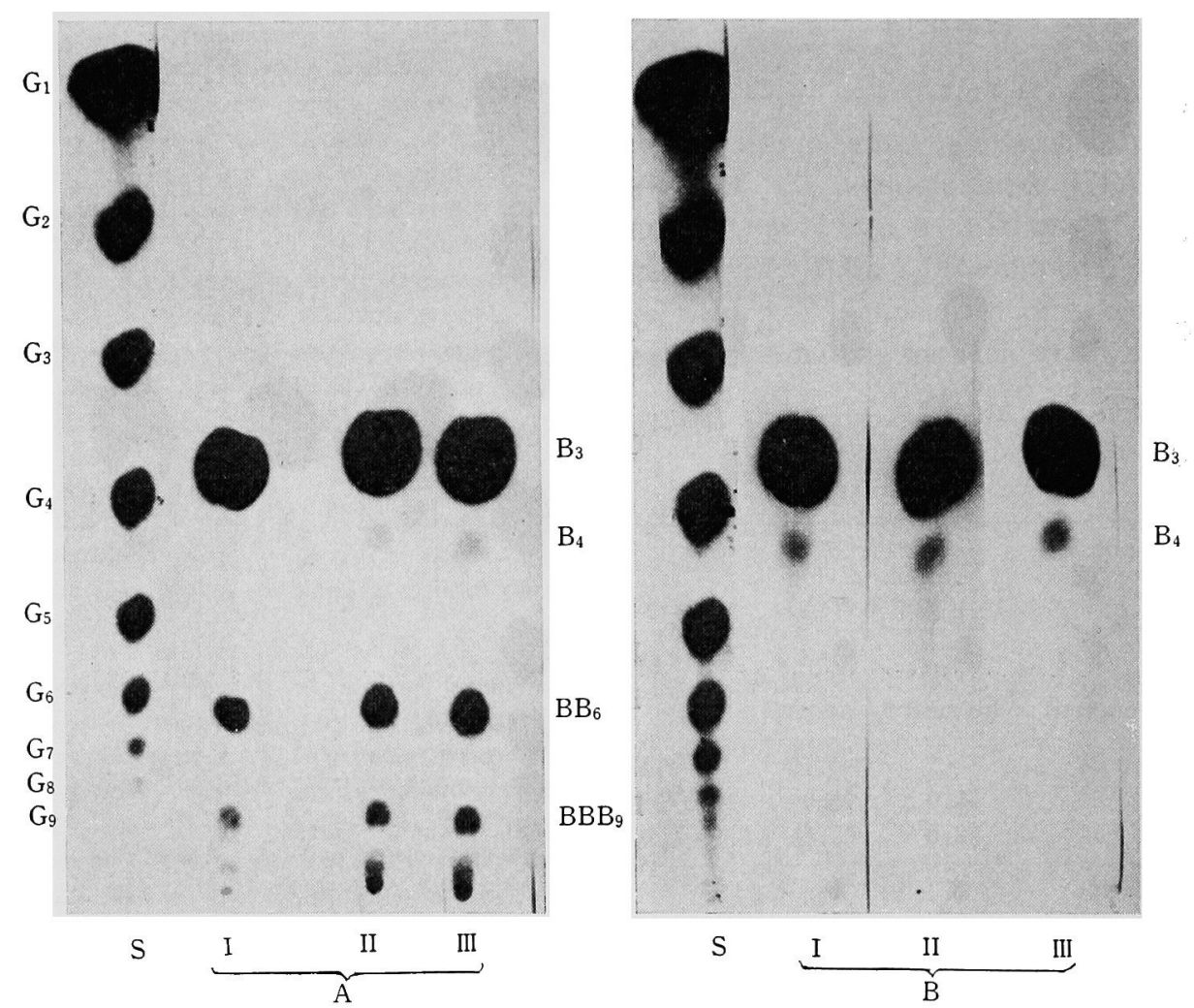

FIG. 3. Hydrolysis of Pullulan by a-1,4-Pullulanase.

A. Paper chromatogram of partially hydrolyzed pullulan ( 5 min reaction).

B. Paper chromatogram of completely hydrolyzed pullulan (120 min reaction). Key: S, standards; $\mathrm{G}_{1} \sim \mathrm{G}_{9}$, glucose $\sim$ maltononaose; I, $P$. pullulans $\mathrm{S}-1$; II, $P$. pullulans OUT 4242; III, P. pullulans IFO 6353.

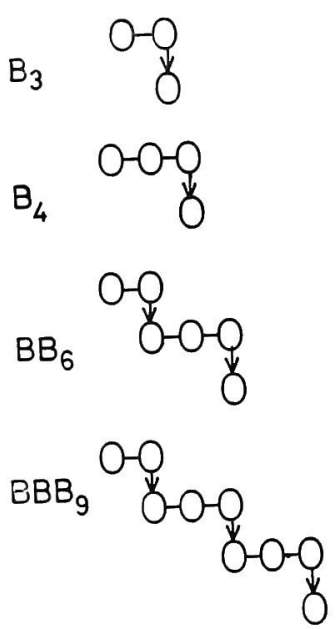

Fig. 4. Structure of Oligosaccharides Formed from Pullulan by $a-1,4-$ Pullulanase Action. Symbols are same as in Fig. 2.
9 as major products with small amounts of tetrasaccharide at early stages, and at complete hydrolysis gave isopanose and a tetrasaccharide supposed to be 6- $a$-maltotriosylglucose, the amounts of which determined as above corresponding to $93 \sim 95 \%$ and $5 \sim 7 \%$ of pullulan, respectively. The hexa- and nonasaccharides $\left(\mathrm{BB}_{6}\right.$ and $\mathrm{BBB}_{9}$ in Fig. 3) appeared at early stages of hydrolysis were further hydrolyzed by the enzyme to isopanose. Thus, their structures were considered to be those given in Fig. 4, as judged from the specificity of the enzyme.

Patterns of enzymatic hydrolysis and the amounts of ultimate products were almost the same for 3 samples of purified pullulan obtained by 3 different strains. 
Table II, Periodate Oxidation Data of CTA-soluble POLYSACCHARIDE FROM $P$. pullulans

\begin{tabular}{ccccc}
\hline $\begin{array}{c}\text { Strains of } \\
\text { organism }\end{array}$ & $\begin{array}{c}\text { Periodate } \\
\text { consumed } \\
\text { (moles per anhydro- } \\
\text { glucose unit) }\end{array}$ & $\begin{array}{c}\text { Formic acid } \\
\text { produced }\end{array}$ & $\begin{array}{c}\text { Ratio of } a-1,6-\text { and } \\
a-1,4 \text {-glucosidic linkages }\end{array}$ \\
\hline S-1 & 1.327 & 0.321 & 32.1 & $6-1,4-(\%)$ \\
IFO 6353 & 1.327 & 0.316 & 31.6 & 68.4 \\
OUT 4242 & 1.329 & 0.290 & 29.0 & 71.0 \\
\hline
\end{tabular}

* Calculated by subtracting $a-1,6(\%)$ from 100 .

\section{Periodate oxidation}

Oxidation of purified pullulan by periodate was complete after $96 \mathrm{hr}$. The amounts of periodate consumed and formic acid produced per anhydroglucose unit, and the ratio of $a-1,6-$ to $a-1,4-$ glucosidic linkages in pullulan calculated therefrom are shown in Table II. As shown in the table, the values were nearly the same for 3 samples of pullulan.

5. Solution viscosity and molecular weight of pullulan, and their variation during cultivation of the organism

The viscosity of aqueous solution of pullulan was measured at $25^{\circ} \mathrm{C}$ with samples obtained from $P$. pullulans $\mathrm{S}-1$ at various culture periods, and values of intrinsic viscosity shown in Table III were obtained. The molecular weight calculated from intrinsic viscosity using a molecular weight-viscosity relationship given by Wallenfels et al. ${ }^{2 /}$ was 170,000 , corresponding to a polymerization degree 1050 , for pullulan obtained after $96 \mathrm{hr}$ culture. Molecular weight of pullulan increased gradually during cultivation period to reach the maximum after $96 \mathrm{hr}$, and then decreased.

Table III. Intrinsic Viscosity of Pullulan Obtained at Various Culture Periods

Strain: Pullularia pullulans S-1.

The concentrations of solutions are expressed in $\mathrm{g} / \mathrm{ml}$.

\begin{tabular}{lcccc}
\hline $\begin{array}{l}\text { Culture periods } \\
\text { (hr) }\end{array}$ & 24 & 48 & 96 & 160 \\
$\begin{array}{l}\text { Intrinsic } \\
\text { viscosity }\end{array}$ & 64 & 70 & 82 & 26 \\
\hline
\end{tabular}

\section{DISCUSSION}

Structure of CTA-soluble polysaccharide, viz., pullulan, produced by $P$. pullulans was studied with chemical and enzymatic methods in the present studies. Three strains of the organism used here produced from sucrose considerably variable amounts of polysaccharides, and the ratio of CTA-soluble to insoluble polysaccharides also varied widely for each strain. But the results of acid and enzymatic hydrolysis, and periodate oxidation on purified pullulan showed that the structure of this fraction of polysaccharide was very similar throughout these strains of organism, consisting mainly of maltotriosyl units joined together with $a$-1,6-glucosidic linkages. Moreover, it was also revealed in enzymatic hydrolysis that the pullulan contained $5 \sim 7 \%$ of maltotetraosyl units. If the pullulan contains $6.6 \%$ of maltotetraosyl units as mentioned by Catley and Whelan, ${ }^{5}$ the ratio of $a-1,6$ - to $a-1,4$-bonds would be $32.7: 67.3$ rather than 33.3: 66.7 expected for polymaltotriose. Then it would be expected on periodate oxidation to consume 1.327 moles periodate and produce 0.327 mole formic acid per anhydroglucose unit. The values were in good agreement with those in Table II within experimental error, and results are in fine coincidence with those in the report of Catley and Whelan.

It is noteworthy that the ratio of maltotriosyl to maltotetraosyl units is almost the same in each strain, in spite of wide variation in the productivity and composition of total polysaccharides. Thus a remarkably high regularity was proved in the structure of 


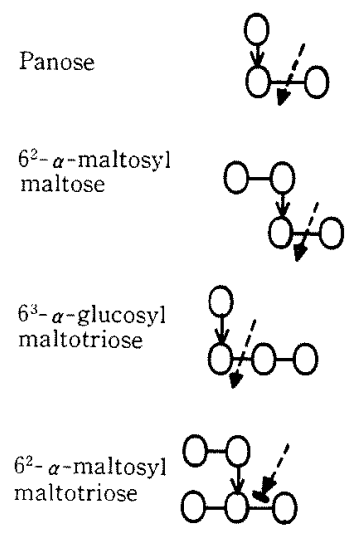

FIG. 5. Action Pattern of $a-1,4-P u l l u l a n a s e$.

Key: O, glucose unit; - $a-1,4$-glucosidic bond; $\downarrow, a-1,6$-glucosidic bond; $x^{\prime}$, point of enzyme attack; $\checkmark$, linkage not attacked by the enzyme.

pullulan in comparison with rather irregularly branched structure of amylopectin or glycogen.

In addition, present results produce an evidence for the complete absence of branched structure in pullulan. Although a linearly connected structure composed of maltotriosyl (and maltotetraosyl) units was assigned to pullulan, the possibility of branching could not be entirely excluded. Here in this paper, all pullulan samples from various strains were shown to be hydrolyzed by a-1,4-pullulanase completely into isopanose and a tetrasaccharide which is assumed to be 6-a-maltotriosylglucose. It was already proved ${ }^{7}$ that the enzyme attacks $a$-1,4-bonds in panose, $6^{3}-a$ glucosylmaltotriose and $6^{2}-a$-maltosylmaltose, but the $a-1,4$-bond is resistant to the enzyme in a structure like $6^{2}$-a-maltosylmaltotriose (cf. Fig. 5). Therefore, if there would be present any branching in the pullulan structure, the hydrolysis by a-1,4-pullulanase could not be complete. The results showing complete hydrolysis of all pullulan samples by the enzyme reported here strongly support the view that there is essentially no possibility of branching in the pullulan structure.

As mentioned in detail above, it is established that the pullulan produced by various strains of $P$. pullulans is uniform in structure, and that there exists no possibility of branching in pullulan structure. The inconsistency of present results and results of Ueda et al..$^{4}$ may be ascribed to possible contamination of other polysaccharides in pullulan preparations employed by the latter authors.

The molecular weight of pullulan increased gradually during the cultivation of the organism to reach the maximum, and then decreased. But the structure of pullulan obtained at early and later stages of cultivation was similar. Concerning the mechanism of polymerization and depolymerization of pullulan during cultivation, nothing is known at present, and the problem must await for future studies.

Acknowledgements. The authors are grateful to Professor Seinosuke Ueda, Kyushu University, for supplying a strain of $P$. pullulans, and to Mr. Kazuo Ebihara for preparations of Aerobacter isoamylase. This study was supported in part by the Scientific Research Grant from the Ministry of Education.

\section{REFERENCES}

1) H. Bender und K. Wallenfels, Biochem. Z., 334, 79 (1961).

2) K. Wallenfels, G. Keilich, G. Bechtler und D. Freudenberger, ibid., 341, 433 (1965).

3) H. O. Bouveng, H. Kiessling, B. Lindberg and J. McKay, Acta Chem. Scand., 16, 615 (1962); idem, ibid., 17, 797 (1963).

4) S. Ueda, K. Fujita, K. Komatsu and Z. Nakashima, Appl. Microbiol., 11, 211 (1963); S. Ueda, Kogyo Kagaku Zasshi, 67, 757 (1964).

5) B. J. Catley and W. J. Whelan, Arch. Biochem. Biophys., 141, 138 (1971).

6) J. E. Scott, "Methods in Biochemical Analysis," Vol. 8, ed. by D. Glick, Interscience Publishers Inc., New York, 1960, p. 145.

7) Y. Sakano, N. Masuda and T. Kobayashi, Agr. Biol. Chem., 35, 971 (1971); Y. Sakano, M. Higuchi and T. Kobayashi, Arch. Biochem. Biophys., 153, 180 (1972).

8) G. O. Aspinall and R. J. Ferrier, Chem. Ind., 1956, 1216.

9) J. E. Hodge and B. T. Hofreiter, "Methods in Carbohydrate Chemistry," Vol. I, ed. by R. L. Whistler and M. L. Wolfrom, Academic Press Inc., New York, 1962, p. 388.

10) N. Nelson, J. Biol. Chem., 153, 375 (1944).

11) M. Somogyl, ibid., 195, 19 (1952).

12) K. Kainuma and D. French, FEBS Letters, 5, 257 (1969).

13) D. French, M. L. Levine, E. Norberg, P. Nordin, J. H. Pazur and G. Wild, J. Am. Chem. Soc., 76, 2387 (1954). 